\title{
Ultra-strong coupling of molecular materials: spectroscopy and dynamics
}

\author{
Jino George, Shaojun Wang, Thibault Chervy, Antoine Canaguier- \\ Durand, Gael Schaeffer, Jean-Marie Lehn, James A. Hutchison,* \\ Cyriaque Genet and Thomas W. Ebbesen*
}

Received 7th October 2014, Accepted 20th October 2014

DOI: $10.1039 / c 4 f d 00197 d$

\begin{abstract}
We report here a study of light-matter strong coupling involving three molecules with very different photo-physical properties. In particular we analyze their emission properties and show that the excitation spectra are very different from the static absorption of the coupled systems. Furthermore we report the emission quantum yields and excited state lifetimes, which are self-consistent. The above results raise a number of fundamental questions that are discussed and these demonstrate the need for further experiments and theoretical studies.
\end{abstract}

\section{Introduction}

One of the advantages of surface plasmon polaritons is their ability to concentrate the electromagnetic field in a very small volume, which in turn can enhance various phenomena and notably light-matter interactions. Therefore it is not surprising that plasmonic structures and micro-cavities have been used interchangeably to provide confined EM fields to induce light-matter strong coupling in molecular materials. Metallic cavities provide some of the strongest field confinements and have the additional feature that both the Fabry-Perot and plasmonic waveguide modes are present. ${ }^{\mathbf{1} 2}$ When molecular materials are placed in a high concentration in such confined fields, hybrid light-matter states, the socalled polaritonic states $\mathrm{P}+$ and $\mathrm{P}-$, are formed with Rabi splittings in the range of 0.1 to $1 \mathrm{eV}$, as illustrated in Fig. 1. When the Rabi splitting is a fraction of the transition energy $\left(\hbar \omega_{M}\right)$, the perturbation of the coupled system is so strong that the energies of the other states of the system are modified. This is the so-called ultra-strong coupling regime where the ground state energy has been predicted to shift $^{3}$ and this has indeed been observed experimentally. ${ }^{4,5}$ In addition, the hybrid light-matter eigen states are in principle delocalized over the optical mode and, upon excitation, quasi-bosonic exciton-polaritons are formed. 
Such features of a strongly coupled system are quite unique and therefore have naturally generated much research activity from single atom-single photon studies to coupling in inorganic semiconductors and organic dyes. ${ }^{6-18}$

While early studies on organic materials focused on the J-aggregates of cyanine dyes ${ }^{18-32}$ due to their sharp and intense absorption peak, a greater variety of molecules are currently being investigated under strong coupling, depending on the scientific targets. ${ }^{33-44}$ For instance, the composite bosonic nature of excitonpolaritons makes them of great interest for thresholdless lasing and other collective effects such as Bose-Einstein condensation. However, such reports remain elusive, being demonstrated only for a system incorporating an anthracene single crystal, ${ }^{33}$ and more recently for fluorinated ladder polymers. ${ }^{34,35} \mathrm{We}$ have been more interested in how the molecular material properties of the coupled system can be modified relative to the bare molecules. Therefore we have chosen other molecules with specific functionalities, such as merocyanine, which is capable of (photo-)switching between two isomers, to demonstrate the effect of those functionalities on chemical reaction rates, work function, phase transitions, and electronic transport. ${ }^{41-45}$ The observation of such effects belies the notion that only a very small percentage of molecules are coupled for each class of molecules. In fact, the stronger the Rabi splitting, the larger the fraction of coupled molecules. ${ }^{5}$

Molecular materials are typically highly disordered and inhomogeneously broadened and this has consequences on the strong coupling, both on the properties of the polaritonic states and on the whole system, as pointed out by Michetti and La Rocca. ${ }^{16}$ In particular, the degree of delocalization of the polaritonic states inside a cavity, together with the fraction of coupled molecules, can be significantly affected. In addition, organic molecules used in strong coupling have typically 100 to 200 normal vibrational modes that also have strong consequences on their properties..$^{32,46,47}$

With vacuum Rabi splittings $\left(\hbar \Omega_{\mathrm{RV}}\right)$ in the order of $1 \mathrm{eV}$, importantly, the coupled system is no longer in the Markovian regime since $\hbar \Omega_{\mathrm{RV}} \gg k_{\mathrm{B}} T{ }^{47}$ As a consequence, the lifetimes of the polaritonic states $\mathrm{P}+$ and $\mathrm{P}-$ cannot be predicted from the lifetimes of the constituents of the system, namely the lifetime of the photon in the cavity and lifetime of the excited state. As we have pointed out elsewhere, ${ }^{32}$ there are numerous examples of coupled systems that are longer lived than their constituents, for instance in the case of molecular excimers and exciplexes.

A large $\hbar \Omega_{\mathrm{RV}}$ can be obtained by using a molecular transition with high absorption cross-sections or transition dipole moments $(d)$ and metallic cavities with small mode volumes $(v)$ since, in the absence of dissipation, the Rabi splitting energy $\left(\hbar \Omega_{\mathrm{R}}\right)$ between the two new hybrid light-matter states is given by: ${ }^{6}$

$$
\hbar \Omega_{\mathrm{R}}=2 E d \sqrt{n_{\mathrm{ph}}+1}=2 \sqrt{\frac{\hbar \omega}{2 \varepsilon_{0} v}} d \sqrt{n_{\mathrm{ph}}+1}
$$

where $E$ is the electric field amplitude of the cavity, $\hbar \omega$ the cavity resonance or transition energy, $\varepsilon_{0}$ the vacuum permittivity, $v$ the mode volume and $n_{\mathrm{ph}}$ the number of photons in the cavity mode. As eqn (1) shows, even when $n_{\mathrm{ph}}$ goes to zero, there remains a finite value for the Rabi splitting $\left(\hbar \Omega_{\mathrm{RV}}\right)$ due to the interaction with the vacuum field. This splitting is itself proportional to the square root 
of the number of molecules in the cavity $\sqrt{N}$, which implies that the $\hbar \Omega_{\mathrm{RV}}$ is proportional to the square root of the molecular concentration $\left(\sqrt{\frac{N}{v}}\right)$ in the optical field.

Crucial for all these studies is a better understanding of the properties of the polaritonic states (e.g. Fig. 1), their dynamics and how they are modified relative to the bare molecules. A greater variety of molecules need to be characterized to look for trends. We have undertaken such a study and, in this report, the photophysical properties of a subset of strongly coupled molecules with very different bare features are compared using both static and fs time-resolved pump-probe spectroscopy. The results raise fundamental questions that will be discussed.

In this paper the three different molecules that were investigated under strong coupling are as follows:

\subsection{TDBC}

The first one is the well-known 1,1'-diethyl-3,3'-bis(4-sulfobutyl)-5, $5^{\prime}, 6,6^{\prime}$-tetrachlorobenzimidazolocarbocyanine (TDBC) in its J-aggregated form. J-aggregates are themselves formed by transition dipole-dipole strong coupling of neighboring molecules, where the symmetry of the aggregates is such that only the transition from the ground state to the lower coupled state is allowed, as illustrated in Fig. 2b. The Stoke shift is characteristically very small $(<10 \mathrm{meV})$.

The Rabi splitting is such that $\mathrm{P}-$ lies below the Frenkel excitonic state involved in the coupling process (Fig. 2a). As a consequence, the energy level of the lower polariton branch lies amongst the higher vibrational states of the ground state manifold. ${ }^{47}$ Thus, even if the polariton state is formed efficiently, it will relax very efficiently by dissipating energy into this bath of non-radiative states. This is despite the fact that polaritons, generated by excitation to $\mathrm{P}-$ or $\mathrm{P}+$, are expected to interact (scatter) very weakly with phonons due to their low effective mass (being quasi-bosons). Recent measurements on TDBC (having $\sim 180$ normal vibration modes) show, indeed, that the non-radiative decay rate constant $k_{\mathrm{nr}}^{\mathrm{P}-}$ of $\mathrm{P}-$ is smaller than the corresponding $k_{\mathrm{nr}}$ of the bare molecule. ${ }^{32}$ Nevertheless, in both cases the non-radiative rate constants are in the order of $10^{12} \mathrm{~s}^{-1}$. This dominates the decay kinetics of $\mathrm{P}-$. It is also the source of the low $\mathrm{P}-$ fluorescence quantum yield, $\Phi_{\mathrm{F}}^{\mathrm{P}-}=\frac{k_{\mathrm{r}}^{\mathrm{P}-}}{k_{\mathrm{nr}}^{\mathrm{P}-}+k_{\mathrm{r}}^{\mathrm{P}-}}$, since the radiative rate

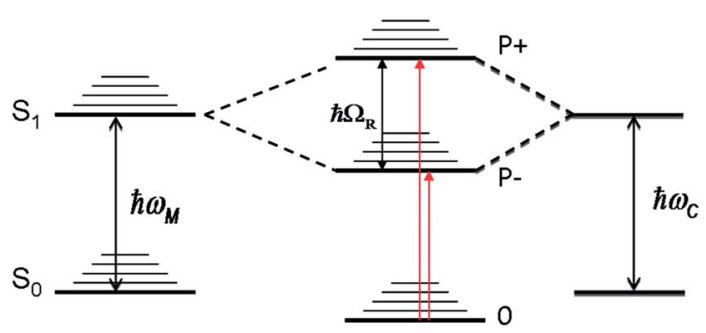

Fig. 1 A schematic diagram of the coupling between a molecular transition ( $\hbar \omega_{M}$ ) and a cavity mode $\left(\hbar \omega_{C}\right)$ giving rise to new hybrid light-matter eigen states $\mathrm{P}+$ and $\mathrm{P}-$. The dispersive nature of these states are not shown. 
<smiles></smiles>

TDBC

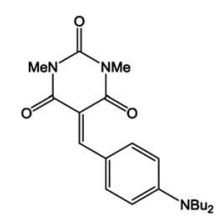

BDAB<smiles></smiles>

Fluorescein

b

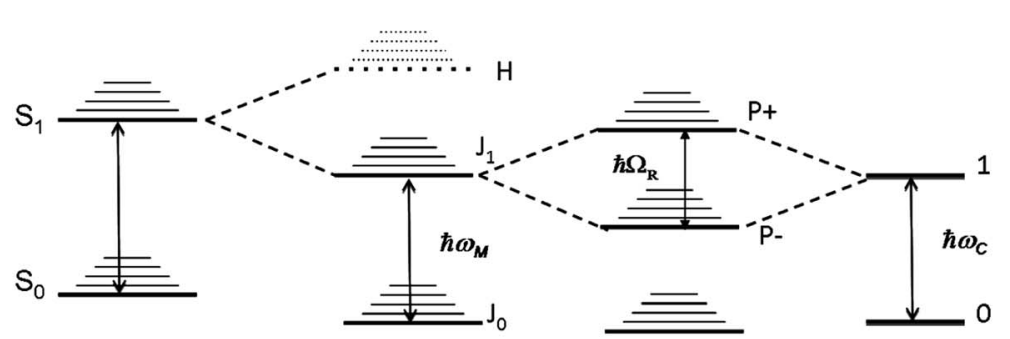

c

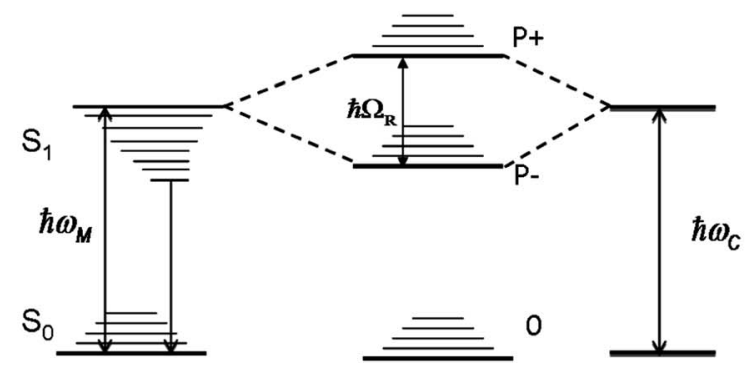

Fig. 2 Molecular structures of the three compounds (a) and the corresponding energy diagrams for TDBC (b) and BDAB (c) upon coupling. The TDBC dipole-dipole coupling gives rise to new states marked $\mathrm{J}$ and $\mathrm{H}$, but for the $\mathrm{J}$-aggregates only the $\mathrm{J}$ state is observed in the absorption spectrum as the transition to $\mathrm{H}$ is forbidden. Fluorescein is coupled schematically as in Fig. 1.

constant $k_{\mathrm{r}}^{\mathrm{P}-}$ has an upper limit for a fully allowed transition in the order of $10^{9} \sim$ $10^{10} \mathrm{~s}^{-1}$. Vibrational relaxation within the excited states occurs on the $100 \mathrm{fs}$ timescale and is characterized by small spectral shifts or sliding during the relaxation. This has also been seen for the $\mathrm{P}-$ of TDBC and it is important that this is not confused with other possible excited decay pathways when observing $\mathrm{P}-$ and $\mathrm{P}+$ at very short time scales. ${ }^{30}$

\subsection{BDAB}

The second molecule is a so-called push-pull molecule, 5-(4-(dibutylamino)benzylidene)-1,3-dimethylpyrimidine-2,4,6(1H,3H,5H)-trione (BDAB), ${ }^{5}$ with an unusually large Stokes shift ( $\sim 560 \mathrm{meV})$. The strong coupling energy level diagram for such molecular systems that undergo electronic energy re-distribution in the excited state, with the associated solvation shell re-distribution, i.e. systems featuring a large Stoke shift, is shown in Fig. 2c. The strong coupling in 
this case involves the transition from the molecular ground state to a higher vibrational state of the excited state manifold (the Franck-Condon transition). As a consequence, the lower $\mathrm{P}$ - polariton branch may lie above, or be isoenergetic with, the relaxed excited state of the bare molecule (see for instance ref. 37). This may have consequences on the dynamics and the decay processes of the coupled system, as has already been shown by Törmä and co-workers. ${ }^{37}$

\subsection{Fluorescein}

Fluorescein, as its name indicates, is a highly fluorescent dye in dilute solutions with a $\Phi_{\mathrm{F}} \simeq 0.9$ and a Stoke shift of $\sim 110 \mathrm{meV}$. It is a representative of standard organic dyes and will strongly couple to the cavity, as illustrated in Fig. 1.

\section{Results}

The strongly coupled samples were prepared by encasing polymer films containing the dyes between two silver films to form an optical cavity (see Experimental section for details). The thickness of the polymer film, and thus the final separation between the $\mathrm{Ag}$ mirrors in the cavity, was chosen to give an optical mode resonant with the absorption maximum of the dye/aggregate (at $590 \mathrm{~nm}$ for TDBC, $470 \mathrm{~nm}$ for BDAB, and $510 \mathrm{~nm}$ for fluorescein). The Q-factors of these cavities are low, typically $\sim 10-20$. Unless specified otherwise, the cavity thicknesses are tuned such that the Rabi splitting occurs at normal incidence $\left(k_{/ /}=0\right)$ where the photon-exciton mixing is $50: 50$.

\subsection{Transition probability to $\mathbf{P}-$}

We start by comparing in Fig. 3a-c the optical absorption and emission (fluorescence) spectra of the Fabry-Perot cavities containing TDBC, fluorescein and $\mathrm{BDAB}$ at normal incidence with their bare spectra. All the absorption spectra are inhomogeneously broadened, even TDBC. The two new absorption peaks corresponding to $\mathrm{P}+$ and $\mathrm{P}-$ (blue solid curves in Fig. 3) have a full width half maximum (FWHM) that is mainly determined by that of the cavity mode, as expected. ${ }^{14}$ It should be noted that for fluorescein the cavity induced splitting is more complex, due to the double peaked absorption of the bare molecule, as seen before for Rhodamine. ${ }^{37}$ The coupling strengths $\left(\hbar \Omega_{\mathrm{RV}}\right)$ for these samples are, respectively, 277, 554 and $720 \mathrm{meV}$ for $\mathrm{TDBC}, \mathrm{BDAB}$ and fluorescein and the corresponding fractions of the transition energy $\left(\hbar \Omega_{\mathrm{RV}} / \hbar \omega_{0}\right)$ are 13,24 and $27 \%$. These fractions, especially the latter two, are sufficiently high that the coupled systems are in the ultra-strong coupling regime, which has consequences on their properties as already discussed. Notice that the $\mathrm{P}-$ fluorescence peak of coupled $\mathrm{BDAB}$ is at higher energy than that of the bare molecule in agreement with the schematic illustration of Fig. 2c. Careful observation of the emission spectra of the coupled systems shows that at least some fluorescence of the bare molecules is always present in these cavities. In other words, some of the molecules are not coupled to the cavity due to the disorder (energetic and orientational) in the system. ${ }^{5,16}$ This uncoupled emission does not disperse with angle and therefore can be easily distinguished from the $\mathrm{P}-$ emission. The apparent Stoke shift of the $\mathrm{P}$ - emission for all three molecules is extremely small or, depending on the conditions of the sample, it sometimes even appears to be slightly blue shifted 


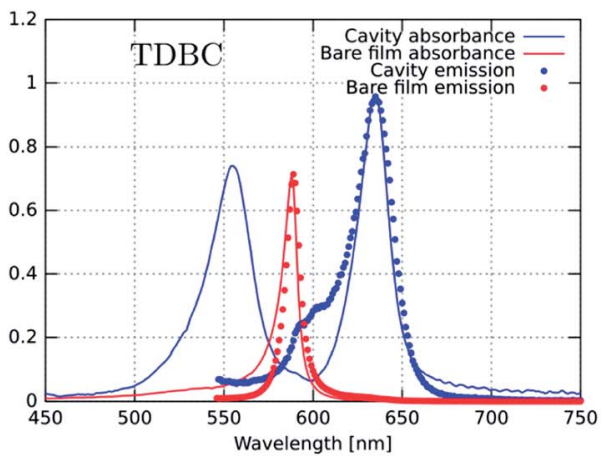

Paper
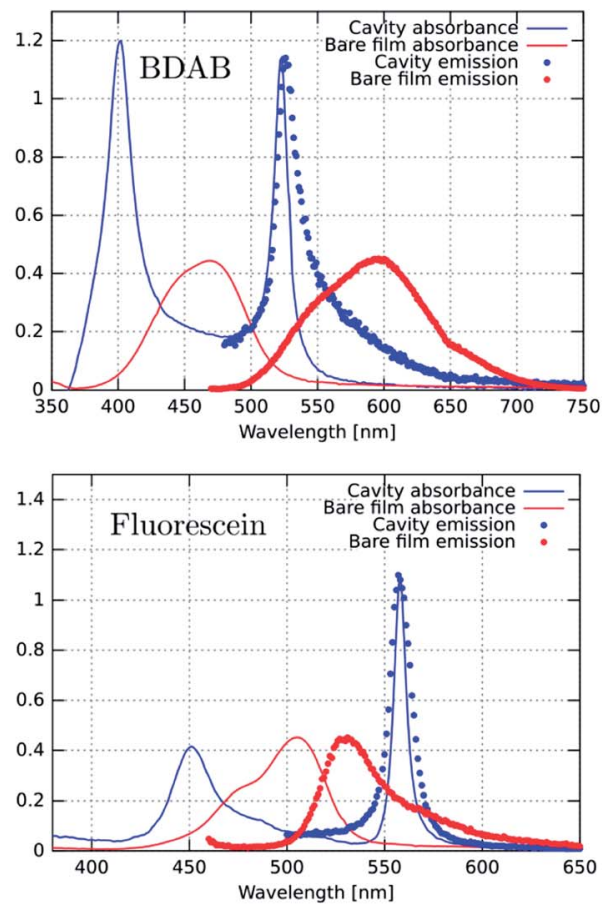

Fig. 3 Absorbance (solid lines) and emission (dotted lines) spectra of the bare molecules (red) and the corresponding coupled systems (blue). The excitation wavelengths were either $530 \mathrm{~nm}$ for TDBC or $450 \mathrm{~nm}$ for BDAB and fluorescein.

relative to absorption..$^{30}$ The blue shifted emission and its origin will be discussed again further on, after the presentation of more results.

Next, we measured the excitation spectra or action spectra associated with the $\mathrm{P}-$ emission. This is a standard technique used in molecular science to understand the origin of the emission and associated processes and we first introduced this for studying strong coupling in an earlier study. ${ }^{30}$ It consists of measuring the emission or fluorescence intensity, $I_{\mathrm{F}}(\lambda)$, at a given wavelength while scanning the excitation wavelength through the absorption spectrum of the sample. Since $I_{\mathrm{F}}(\lambda)$ $\propto \Phi_{\mathrm{F}} \sigma_{\mathrm{A}}(\lambda) I_{0}(\lambda) c=\Phi_{\mathrm{F}} I_{\mathrm{A}}(\lambda)$, where $\sigma_{\mathrm{A}}(\lambda)$ is the absorption cross section of the molecule, $c$ is the molecular concentration and $I_{0}(\lambda)$ is the incident light intensity, 
one would expect that when $I_{0}$ is normalized then $I_{\mathrm{F}}(\lambda)$ will be proportional to the light absorbed, $I_{\mathrm{A}}(\lambda)$. Therefore, by just plotting $I_{\mathrm{F}}$ at a given $\lambda$ while scanning the normalized excitation, $I_{0}(\lambda)$, the shape obtained should reflect the absorbance of the states contributing to the emission process.

Fig. 4a, $\mathrm{c}$ and $\mathrm{d}$ show the excitation spectra of the three molecules recorded for their $\mathrm{P}-$ emission and these are compared to their absorbance spectra. The first striking feature is the relative contribution of $\mathrm{P}+$ and $\mathrm{P}-$ to the emission of $\mathrm{P}-$ (here all the excitation spectra have been normalized to the $\mathrm{P}+$ absorbance peak). It thus appears that the direct excitation of the $\mathrm{P}$ - peak contributes very little to the $\mathrm{P}-$ emission, while when exciting to $\mathrm{P}+$ or the uncoupled molecules it rapidly relaxes to $\mathrm{P}-$, where emission can occur. This relaxation to $\mathrm{P}-$ can occur via energy transfer between the coupled and the uncoupled molecular reservoirs as well as by rapid internal vibrational relaxation (IVR). The latter is a standard feature of molecules, with their very large number of normal modes and their overtones that provide a continuum of sublevels between the various states. It is interesting to note that the ratio of $\mathrm{P}-/ \mathrm{P}+$ excitation peaks is the smallest for TDBC $(\sim 0.1)$ and the largest for BDAB $(\sim 0.5)$.

This raises the question of whether the direct excitation into the $\mathrm{P}-$ static absorption peak actually populates $\mathrm{P}$ - or not. To answer this question we also recorded the excitation spectrum of pump-probe experiments by looking at the

a

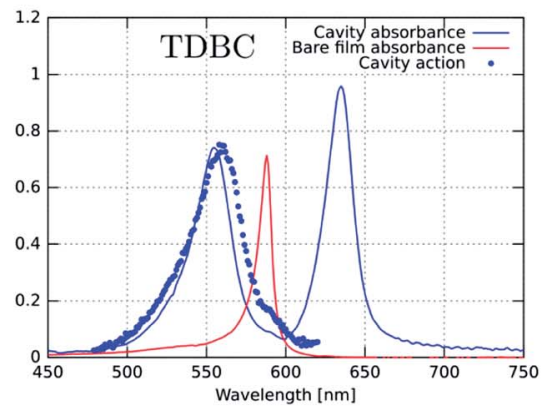

$\mathrm{c}$

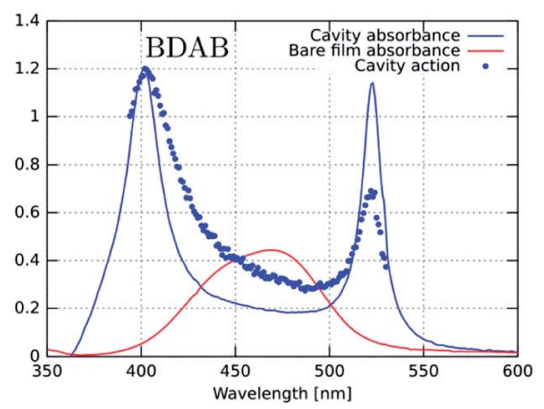

b

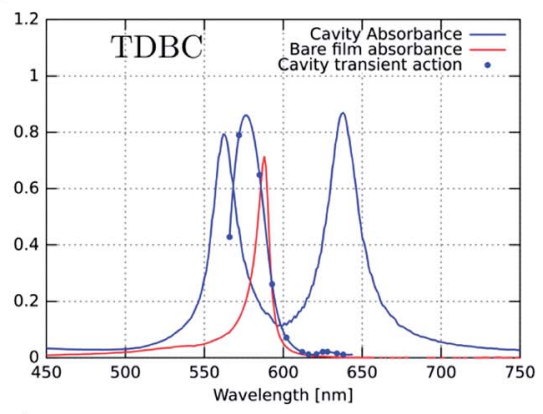

d

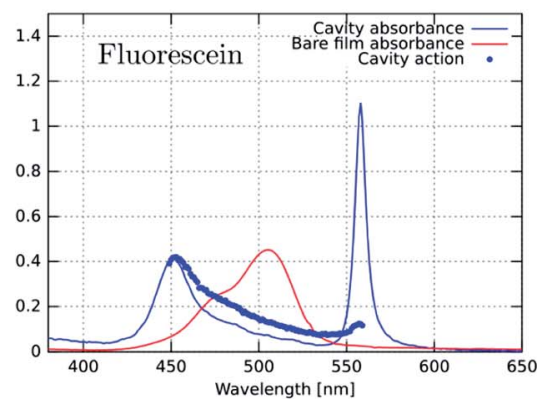

Fig. 4 Excitation or action spectra (blue dots) recorded at normal incidence for $\mathrm{P}$ emission for (a) TDBC (at $680 \mathrm{~nm}$ ), (c) BDAB (at $540 \mathrm{~nm}$ ) and (d) Fluorescein (at $576 \mathrm{~nm}$ ) and (b) for $\mathrm{P}-$ transient absorption of TDBC. These spectra are compared to the bare molecule absorbance spectra (solid red lines) and the strongly coupled system absorbance spectra (solid blue lines) of the same samples. 
intensity of the transient absorption spectrum of $\mathrm{P}-$, proportional to the population of $\mathrm{P}-$, as a function of laser pump wavelength. This was possible to do for TDBC since our 150 fs-laser could be tuned to cover the static absorption peaks. The resulting spectrum is plotted in Fig. $4 \mathrm{~b}$ and it has very similar features to the fluorescence excitation spectrum (Fig. 4a). Again, the direct excitation of $\mathrm{P}-$ appears not to be allowed. Interestingly, the $\hbar \Omega_{\mathrm{RV}}$ deduced from the excitation spectra appears to be smaller than the static absorption spectrum indicates. This is probably the origin of the blue-shifted emission relative to the static absorption that is sometimes observed.

To try to understand the origin of these observations, the cavities were detuned and the fluorescence excitation spectra were recorded. For fluorescein, the detuning of the cavity to higher energies ( $c a .60 \mathrm{meV}$ ) seems to enhance direct $\mathrm{P}-$ excitation, as shown in Fig. 5a (red curve). This could be explained by the higher exciton content of the $\mathrm{P}-$ branch. However, in the case of BDAB (Fig. 5b), the reverse tendency is seen, i.e. detuning to lower energies enhances the relative contribution of $\mathrm{P}-$ to the excitation spectrum. Taken together with the results of Fig. 4, it appears that the internal vibronic structure of the molecule plays an important role and also possibly the excited state rearrangement involved in the Stoke shift.
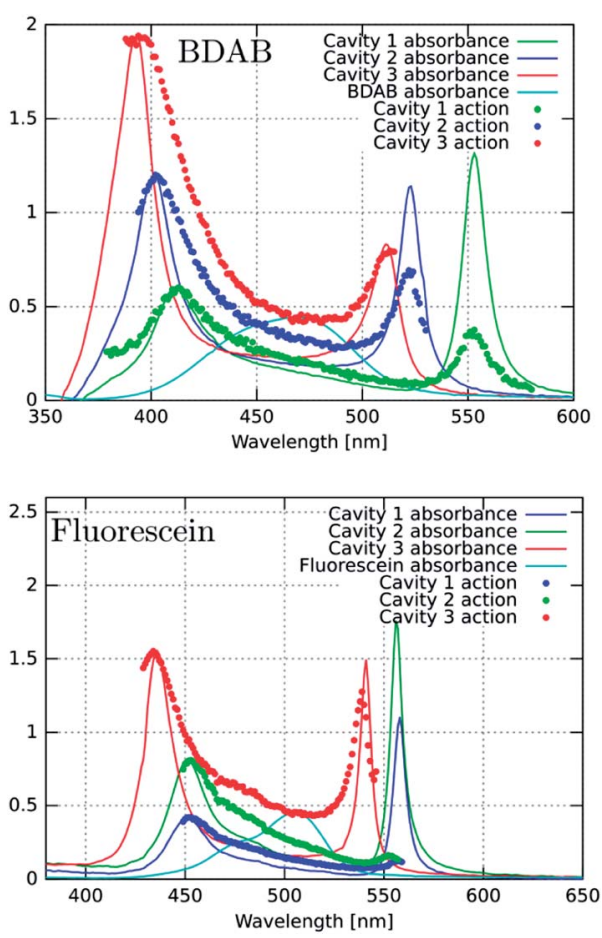

Fig. 5 Excitation spectra (dotted lines) for BDAB and fluorescein for detuned cavities (red and green curves) compared to the tuned cavities (blue curves) and compared to the corresponding absorbance spectra of the coupled systems and the bare molecules (light blue). The emission detection wavelengths for BDAB are $600 \mathrm{~nm}$ (red curve), $540 \mathrm{~nm}$ (blue curve) and $525 \mathrm{~nm}$ (green curve). For fluorescein these are $556 \mathrm{~nm}$ (red curve), $570 \mathrm{~nm}$ (green curve) and $576 \mathrm{~nm}$ (blue curve). 


\subsection{Lifetime of $\mathbf{P}$}

The transient spectra of the three strongly coupled systems are shown in Fig. 6, together with their decay kinetics. The spectra do not evolve over the decay, indicating that they are dominated by a single transient species. The decays are not single exponentials due to the heterogeneities of the samples at the high concentrations that were used to induce strong coupling. The angle dependence of the decay was also measured for TDBC and was found to be invariant, indicating that the lifetime is not dependent on probed k-vector. This indicates that there is no bottle-neck along the P- branch shown in Fig. 6 for this sample. Such decay bottle-necks have been seen in other conditions and samples for strongly coupled TDBC. ${ }^{24}$

Table 1 below summarizes the dynamical data for the strongly coupled molecules. It first compares the observed half-lives measured by transient absorption with those of the 1 /linewidth of the $\mathrm{P}-$ resonance and the experimental radiative lifetimes, as well as the predicted ones derived from absorbance peaks of $\mathrm{P}$ - using the Bowen and Wokes approximation. ${ }^{48}$ It is notable that these two standard methods for estimating lifetimes give such very different values. The measured and calculated quantum yields for TDBC and fluorescein are in very good agreement, considering all the experimental uncertainty, while for BDAB the experimental value is an order of magnitude higher. The latter is not surprising
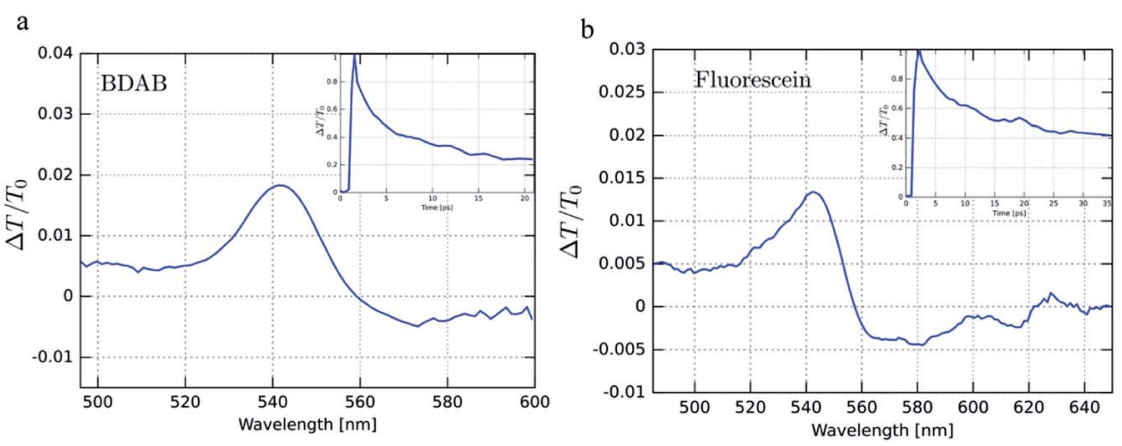

$\mathrm{c}$

d
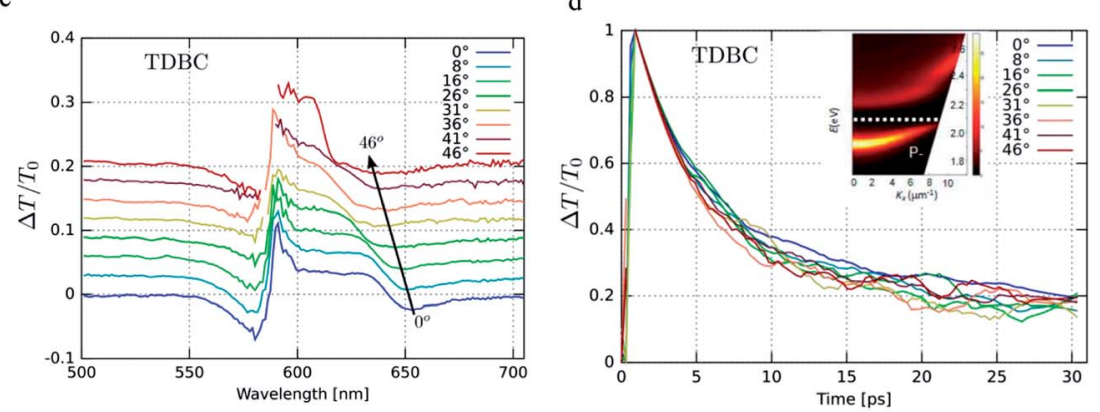

Fig. 6 (a), (b) and (c) Transient pump-probe spectra with the corresponding decays as inserts for BDAB and fluorescein. In the case of TDBC, the angular dependence of the transient spectra is shown in (c) and the corresponding decays in (d). The insert in (d) shows the dispersion of the static absorption of TDBC in the cavity. BDAB and fluorescein were pumped at $400 \mathrm{~nm}$ and TDBC at $585 \mathrm{~nm}$. 
Table 1

\begin{tabular}{|c|c|c|c|}
\hline & TBDC & $\mathrm{BDAB}$ & Fluorescein \\
\hline $\begin{array}{l}1^{\text {st }} \text { half life } \\
\text { (transient absorption) }\end{array}$ & $4.3 \mathrm{ps}$ & $3.5 \mathrm{ps}$ & $30 \mathrm{ps}$ \\
\hline $\begin{array}{l}\text { Cavity lifetime } \\
\text { (1/linewidth) }\end{array}$ & $2 \mathrm{fs}$ & $5 \mathrm{fs}$ & $14 \mathrm{fs}$ \\
\hline $\begin{array}{l}\text { Radiative lifetime } \\
\text { (predicted value) }\end{array}$ & $0.2 \mathrm{~ns}(0.4 \mathrm{~ns})$ & $1 \mathrm{~ns}(0.1 \mathrm{~ns})$ & $3 \mathrm{~ns}(1.5 \mathrm{~ns})$ \\
\hline $\begin{array}{l}\text { Quantum yield } \\
\text { (predicted value) }\end{array}$ & $1 \times 10^{-2}\left(2 \times 10^{-2}\right)$ & $4 \times 10^{-2}\left(3.5 \times 10^{-3}\right)$ & $2 \times 10^{-2}\left(1 \times 10^{-2}\right)$ \\
\hline
\end{tabular}

considering the fact that the emission is not from the Franck-Condon transition but due instead to an intra-molecular charge transfer state being at the origin of the large Stoke shift of the bare molecule. Thus, the Bowen and Wokes approximation is not the best model for such a molecule.

\section{Discussion and conclusions}

Conspicuous from the above excitation spectral experiments is the fact that the static absorption peak due to $\mathrm{P}$ - (in Fig. 3-5) does not readily lead to the generation of $\mathrm{P}-$ emission or even to the $\mathrm{P}-$ transient absorption. We have shown this excitation behaviour before for TDBC and it has since been confirmed by others. $^{24}$ This finding is surprising since one would expect that the static absorption peak of $\mathrm{P}$ - would lead directly to populating $\mathrm{P}-$ and subsequent emission. This is an important issue since it is a standard assumption that the emission in the spectral region of $\mathrm{P}$ - indeed comes from a corresponding state.

One could take a very naïve view to explain the above results from a purely classical interpretation. For instance, the shape of $\mathrm{P}-$ emission spectra can be very easily reproduced by taking the emission of the bare molecules filtered by the transmission properties of the cavity (as can be seen in Fig. 3). The excitation spectrum would therefore be dominated by the spectral regions where the bare molecule absorbs but enhanced by the modal properties of the cavity (as in Fig. 4). This is similar to the treatment of strong coupling by Zhu et al. where it is not necessary to assume the formation of hybrid light-matter states by vacuum Rabi splitting. ${ }^{11}$ In this classical vision, the lifetimes of the $\mathrm{P}-$ measured by fluorescence or by transient absorption spectroscopy would be those of the bare molecule perturbed by the strong interaction with the confined electromagnetic environment of the cavity.

Nevertheless, the observation of novel and modified properties in strongly coupled systems argues strongly for the quantum interpretation and the existence of hybrid light-matter states. These include coherent emission over large distances, ${ }^{23}$ condensation of polariton, ${ }^{34,35}$ polariton lasing ${ }^{33}$ and modification of such properties as chemical rates, ${ }^{42}$ work-function ${ }^{43}$ and conductivity, ${ }^{45}$ amongst others. The significant $\mathrm{P}-$ emission quantum yields also argue against a simple cavity filter effect for the fluorescence. Therefore there is a need to understand why the direct excitation of $\mathrm{P}-$ is a very inefficient way of populating the state, 
despite the 50:50 photonic-exciton mixing for both $\mathrm{P}+$ and $\mathrm{P}-$ in our experiments.

One possibility is that the transition probability is very weak despite the strong static absorption. This immediately raises the question of what is at the origin of the static absorption. One unique characteristic of these organic coupled systems is the large inhomogeneous broadening which, coupled to the cavity, could generate dissipation without populating $\mathrm{P}-$. This would leave indirect excitation via higher energy states involving energy transfer and IVR as the most efficient means of then populating the $\mathrm{P}-$ state. However, as we discussed previously, ${ }^{32}$ the recorded $\mathrm{P}$ - emission quantum efficiencies are only compatible with a lifetime in the picosecond regime, as measured for $\mathrm{P}-$ both in fluorescence and transient absorption. It is important to note that this is for a most favourable $k_{\mathrm{r}}$ corresponding to a highly allowed transition. If the transition to $\mathrm{P}-$ is only weakly allowed, then $\Phi_{\mathrm{F}}^{\mathrm{P}-}$ should be much smaller, unless the strongly coupled system boosts the emission for an otherwise weakly allowed transition.

Another conundrum for us in these systems is also related to lifetimes. From the FWHM of the $\mathrm{P}-$ and $\mathrm{P}+$ absorption, principally determined by the Q-factor of the cavity, ${ }^{14}$ one derives a lifetime of $\sim 10$ fs. If one integrates over the same absorbance peak to derive the radiative lifetime, one obtains a lifetime three orders of magnitude longer. ${ }^{32}$ These represent two very different approaches, one optical and one molecular, to the same issue.

In the spirit of the Faraday discussions, we have shown here, through experiments, some of the open questions related to light-matter strong coupling involving molecular materials. Clearly more experiments and theory that can handle such complex materials and the particularities associated with individual molecular structures need to be developed to elucidate these strongly interacting systems. Only then will the full potential of using a confined electromagnetic environment to control material and molecular properties be realized.

\section{Experimental}

All the steady state measurements were performed in reflective cavities, in which the Ag mirror deposited on the glass was $200 \mathrm{~nm}$ thick, and the top mirror was 30 $\mathrm{nm}$ thick. For pump-probe measurements, experiments were performed in transmission with cavities in which both mirrors were $30 \mathrm{~nm}$ thick. The bottom Ag film was sputtered on borosilicate glass, followed by spin-coating of the molecules dispersed in a polymer to form a thin film, and then sputtering the second $\mathrm{Ag}$ film on top. TDBC films were spin-cast from aqueous solution (Milli-Q) containing $0.5 \%$ TDBC and 5\% poly(vinyl) alcohol (PVA, M.W. 205 000) by weight. Fluorescein films were cast from aqueous solutions of $1 \%$ Fluorescein disodium salt and $2 \%$ PVA by weight. BDAB films were cast from toluene solutions of $0.5 \%$ BDAB and 1\% poly(methyl methacrylate) (PMMA, M.W. 120 000) by weight. Toluene (Sigma Aldrich), PVA (FLUKA), PMMA (Sigma Aldrich), fluorescein disodium salt (Exciton) and TDBC (Few Chemicals) were obtained commercially and used without further purification. BDAB, 5-(4-(dibutylamino)benzylidene)1,3-dimethylpyrimidine-2,4,6(1H,3H,5H)-trione, was synthesized as follows. 4dibutylaminobenzaldehyde (196 mg, $840 \mu \mathrm{mol}, 1.00$ eq.) and 1,3-dimethylpyrimidine-2,4,6(1H,3H,5H)-trione $(131 \mathrm{mg}, 840 \mu \mathrm{mol}, 1.00 \mathrm{eq}$.) were dissolved in $5 \mathrm{~mL}$ of ethanol. The resulting mixture was refluxed for $3 \mathrm{~h}$. After cooling the 
precipitate was filtered off and washed with $10 \mathrm{~mL}$ of $\mathrm{H}_{2} \mathrm{O}, 10 \mathrm{~mL}$ of ethanol and $10 \mathrm{~mL}$ of $n$-pentane. The yellow solid was dried in vacuum to give the product in quantitative yield (312 mg). 1H NMR (400 MHz, CDCl3, $\delta / \mathrm{ppm}): 0.98(\mathrm{t}, J=7.3 \mathrm{~Hz}$, $6 \mathrm{H}), 1.45-1.32(\mathrm{~m}, 4 \mathrm{H}), 1.69-1.57(\mathrm{~m}, 4 \mathrm{H}), 3.44-3.37(\mathrm{~m}, 10 \mathrm{H}), 6.66(\mathrm{~d}, J=8.98 \mathrm{~Hz}$, 2H), 8.36-8.41 (d, $J=9.23 \mathrm{~Hz}, 3 \mathrm{H}$ ). ESI-MS: $m / z: 360.3$ (100\%). HRMS (ESI, $m / z)$ : calculated for $\mathrm{C}_{21} \mathrm{H}_{29} \mathrm{~N}_{3} \mathrm{NaO}_{3} 394.210$; found 394.210.

Steady-state transmission and reflection spectra were taken on a Shimadzu UV3101 spectrometer. Steady-state fluorescence spectra were taken with a Horiba Jobin Yvon-Spex Fluorolog-3 fluorimeter. Pump-probe experiments were carried out with narrow band (linewidth $=6 \mathrm{~nm}$ ), 150 fs-pulse tunable SpectraPhysics laser system. The spectra were recorded using low-energy $\left(<100 \mu \mathrm{J} \mathrm{cm}^{-2}\right)$ pumping pulses to avoid spurious effects.

\section{Acknowledgements}

The authors acknowledge support from the ERC (Grants 227557), the International Center for Frontier Research in Chemistry (icFRC, Strasbourg), the ANR Equipex "Union" (ANR-10-EQPX-52-01), the Labex NIE projects (ANR-11-LABX0058_NIE), CSC (ANR-10-LABX-0026_CSC) and USIAS within the Investissement d'Avenir program ANR-10-IDEX-0002-02.

\section{References}

1 S. Kéna-Cohen, S. A. Maier and D. D. C. Bradley, Adv. Opt. Mater., 2013, 1, 827.

2 E. N. Economou, Phys. Rev., 1969, 182, 539.

3 C. Ciuti, G. Bastard and I. Carusotto, Phys. Rev. B: Condens. Matter Mater. Phys., 2005, 72, 115303.

4 P. Forn-Díaz, J. Lisenfeld, D. Marcos, J. J. García-Ripoll, E. Solano, C. J. P. M. Harmans and J. E. Mooij, Phys. Rev. Lett., 2010, 105, 237001.

5 A. Canaguier-Durand, E. Devaux, J. George, Y. Pang, J. A. Hutchison, T. Schwartz, C. Genet, N. Wilhelms, J.-M. Lehn and T. W. Ebbesen, Angew. Chem., Int. Ed., 2013, 52, 10533.

6 S. Haroche and D. Kleppner, Phys. Today, 1989, 42, 24.

7 R. Houdré, Phys. Status Solidi B, 2005, 242, 2167.

8 P. Snoke and D. Littlewood, Phys. Today, 2010, 63, 42.

9 Y. Kaluzny, P. Goy, M. Gross, J. M. Raimond and S. Haroche, Phys. Rev. Lett., 1983, 51, 1175.

10 G. Rempe, H. Walther and N. Klein, Phys. Rev. Lett., 1987, 58, 353.

11 Y. Zhu, D. J. Gauthier, S. E. Morin, Q. Wu, H. J. Carmichael and T. W. Mossberg, Phys. Rev. Lett., 1990, 64, 2499.

12 R. J. Thompson, G. Rempe and H. J. Kimble, Phys. Rev. Lett., 1992, 68, 1132.

13 C. Weisbuch, M. Nishioka, A. Ishikawa and Y. Arakawa, Phys. Rev. Lett., 1992, 69, 3314.

14 R. Houdré, R. P. Stanley and M. Ilegems, Phys. Rev. A, 1996, 53, 2711.

15 F. Tassone, C. Piermarocchi, V. Savona, A. Quattropani and P. Schwendimann, Phys. Rev. B: Condens. Matter Mater. Phys., 1997, 56, 7554.

16 P. Michetti and G. C. La Rocca, Phys. Rev. B: Condens. Matter Mater. Phys., 2005, 71, 115320. 
17 K. G. Lagoudakis, M. Wouters, M. Richard, A. Baas, I. Carusotto, R. Andre, L. E. S. I. Dang and B. Deveaud-Pledran, Nat. Phys., 2008, 4, 706.

18 D. G. Lidzey, D. D. C. Bradley, M. S. Skolnick, T. Virgili, S. Walker and D. M. Whittaker, Nature, 1998, 395, 53.

19 P. A. Hobson, W. L. Barnes, D. G. Lidzey, G. A. Gehring, D. M. Whittaker, M. S. Skolnick and S. Walker, Appl. Phys. Lett., 2002, 81, 3519.

20 Y. Sugawara, T. A. Kelf, J. J. Baumberg, M. E Abdelsalam and P. N. Bartlett, Phys. Rev. Lett., 2006, 97, 266808.

21 N. T. Fofang, N. K. Grady, Z. Fan, A. O. Govorov and N. J. Halas, Nano Lett., 2011, 11, 1556.

22 J. R. Tischler, M. Scott Bradley, Q. Zhang, T. Atay, A. Nurmikko and V. Bulović, Org. Electron., 2007, 8, 94.

23 S. Aberra Guebrou, C. Symonds, E. Homeyer, J. C. Plenet, Y. N. Gartstein, V. M. Agranovich and J. Bellessa, Phys. Rev. Lett., 2012, 108, 066401.

24 D. M. Coles, R. T. Grant, D. G. Lidzey, C. Clark and P. G. Lagoudakis, Phys. Rev. B: Condens. Matter Mater. Phys., 2013, 88, 121303(R).

25 P. Vasa, W. Wang, R. Pomraenke, M. Lammers, M. Maiuri, C. Manzoni, G. Cerullo and C. Lienau, Nat. Photonics, 2013, 7, 128.

26 G. Zengin, G. Johansson, P. Johansson, T. J. Antosiewicz, M. Käll and T. Shegai, Sci. Rep., 2013, 3, 3074.

27 Y.-W. Hao, H.-Y. Wang, Y. Jiang, Q.-D. Chen, K. Ueno, W.-Q. Wang, H. Misawa and H.-B. Sun, Angew. Chem., Int. Ed., 2011, 123, 7970.

28 J. Dintinger, S. Klein, F. Bustos, W. L. Barnes and T. W. Ebbesen, Phys. Rev. B: Condens. Matter Mater. Phys., 2005, 71, 035424.

29 A. Salomon, C. Genet and T. W. Ebbesen, Angew. Chem., Int. Ed., 2009, 48, 8748.

30 T. Schwartz, J. A. Hutchison, J. Léonard, C. Genet, S. Haacke and T. W. Ebbesen, ChemPhysChem, 2013, 14, 125.

31 A. Salomon, S. Wang, J. A. Hutchison, C. Genet and T. W. Ebbesen, ChemPhysChem, 2013, 14, 1882.

32 S. Wang, T. Chervy, J. George, J. A. Hutchison, C. Genet and T. W. Ebbesen, J. Phys. Chem. Lett., 2014, 5, 1433.

33 S. Kéna-Cohen and S. R. Forrest, Nat. Photonics, 2010, 4, 371.

34 J. D. Plumhof, T. Stoferle, L. J. Mai, U. Scherf and R. F. Mahrt, Nat. Mater., 2014, 13, 248.

35 K. S. Daskalakis, S. A. Maier, R. Murray and S. Kéna-Cohen, Nat. Mater., 2014, 13, 271.

36 M. A. Koponen, U. Hohenester, T. K. Hakala and J. J. Toppari, Phys. Rev. B: Condens. Matter Mater. Phys., 2013, 88, 085425.

37 T. K. Hakala, J. J. Toppari, A. Kuzyk, M. Pettersson, H. Tikkanen, H. Kunttu and P. Törmä, Phys. Rev. Lett., 2009, 103, 053602.

38 W. Ni, Z. Yang, H. Chen, L. Li and J. Wang, J. Am. Chem. Soc., 2008, 130, 6692.

39 A. Berrier, R. Cools, C. Arnold, P. Offermans, M. Crego-Calama, S. H. Brongersma and J. Gómez-Rivas, ACS Nano, 2011, 5, 6226.

40 F. Valmorra, M. Bröll, S. Schwaiger, N. Welzel, D. Heitmann and S. Mendach, Appl. Phys. Lett., 2011, 99, 051110.

41 T. Schwartz, J. A. Hutchison, C. Genet and T. W. Ebbesen, Phys. Rev. Lett., 2011, 106, 196405. 
42 J. A. Hutchison, T. Schwartz, C. Genet, E. Devaux and T. W. Ebbesen, Angew. Chem., Int. Ed., 2012, 51, 1592.

43 J. A. Hutchison, A. Liscio, T. Schwartz, A. Canaguier-Durand, C. Genet, V. Palermo, P. Samori and T. W. Ebbesen, Adv. Mater., 2013, 25, 2481.

44 S. J. Wang, A. Mika, J. A. Hutchison, C. Genet, A. Jouaiti, M. W. Hosseini and T. W. Ebbesen, Nanoscale, 2014, 6, 7243.

45 E. Orgiu, J. George, J. A. Hutchison, E. Devaux, J.-F. Dayen, B. Doudin, F. Stellacci, C. Genet, P. Samorì and T. W. Ebbesen, 2014, arXiv:1409.1900 [cond-mat.mtrl-sci].

46 J. A. Ćwik, S. Reja, P. B. Littlewood and J. Keeling, EPL, 2014, 105, 47009.

47 A. Canaguier-Durand, C. Genet, A. Lambrecht, T. W. Ebbesen and S. Reynaud, 2013, arXiv:1307.8378 [cond-mat.mes-hall].

48 J. B. Birks, Photophysics of Aromatic Molecules, Wiley-Interscience, 1970. 